\title{
Safety and Efficacy of Low-Volume Preparation in the Elderly: Oral Sulfate Solution on the Day before and Split-Dose Regimens (SEE SAFE) Study
}

Min Seob Kwak ${ }^{1}$, Jae Myung Cha ${ }^{1}$, Hyo-Joon Yang ${ }^{2}$, Dong II Park ${ }^{2}$, Kyeong Ok Kim ${ }^{3}$, Jun Lee ${ }^{4}$, Jeong Eun Shin ${ }^{5}$, Young-Eun $\mathrm{Joo}^{6}$, Jongha Park ${ }^{7}$, Jeong-Sik Byeon ${ }^{8}$, Hyun Gun Kim ${ }^{9}$, Intestinal Cancer Study Group of the Korean Association for the Study of Intestinal Diseases (KASID)

${ }^{1}$ Department of Internal Medicine, Kyung Hee University School of Medicine, Seoul, ${ }^{2}$ Department of Internal Medicine, Sungkyunkwan University College of Medicine, Suwon, ${ }^{3}$ Department of Internal Medicine, Yeungnam University College of Medicine, Daegu, ${ }^{4}$ Department of Internal Medicine, Chosun University College of Medicine, Gwangju, ${ }^{5}$ Department of Internal Medicine, Dankook University College of Medicine, Cheonan, ${ }^{6}$ Department of Internal Medicine, Chonnam National University Medical School, Gwangju, ${ }^{7}$ Department of Internal

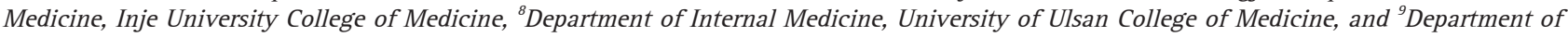
Internal Medicine, Soonchunhyang University College of Medicine, Seoul, Korea

Background/Aims: The use of a low-volume bowel cleansing agent is associated with a greater willingness to undergo repeat colonoscopy. Oral sulfate solution (OSS) is a recently approved low-volume agent; however, its efficacy and safety in the elderly population remain unclear. We aimed to evaluate the efficacy, safety, and acceptability of the OSS preparation, in comparison to those of a standard polyethylene glycol (PEG; 4 L) preparation, in elderly patients. Methods: A multicenter, randomized, investigator-blinded study was conducted. Participants were randomized to receive OSS or 4-L PEG with a split-dose regimen. Bowel cleansing efficacy was assessed using the Boston Bowel Preparation Scale (BBPS). Acceptance, satisfaction, and preparation-related symptoms were recorded. Additionally, blood parameters were analyzed for electrolyte abnormalities and nephrotoxicity. Results: A total of 193 patients were analyzed. No group differences in overall bowel cleansing efficacy were observed, with "adequate" preparations achieved in 95.9\% (93/97) and $94.8 \%$ (91/96) of patients in the OSS and 4L PEG groups, respectively $(p=0.747)$. However, mean BBPS scores for the entire $(p=0.010)$ and right colon $(p=0.001)$ were significantly higher in the OSS group than in the 4-L PEG group. The severity of clinical adverse events and frequency of acute kidney injury were similarly low, and no clinically meaningful electrolyte changes were identified. Self-reported scores regarding amount $(p<0.001)$ and feeling $(p=0.007)$, as well as overall satisfaction ( $p=0.001$ ) and willingness to repeat the preparation $(92.8 \%$ vs $67.7 \%, p<0.001)$, were significantly better in the OSS group than in the 4-L PEG group. Conclusions: In elderly individuals, OSS with a split-dose regimen has greater acceptability and comparable efficacy in bowel cleansing compared to 4-L PEG. (Clinical trials registration number: NCT03112967) (Gut Liver 2019;13:176-182)

Key Words: Colonoscopy; Cathartics; Safety; Treatment outcome; Aged

\section{INTRODUCTION}

The colorectal cancer (CRC) incidence rate increases steadily with age. ${ }^{1,2}$ Colonoscopy is widely endorsed as a screening modality to reduce CRC incidence and mortality. ${ }^{3-6}$ Proper bowel preparation is critical for an effective colonoscopy; however, bowel preparations are often considered by patients to be the most burdensome aspect of colonoscopy ${ }^{7-10}$ Furthermore, age influenced the adequacy of bowel preparation, and poor bowel preparation occurred in up to $57 \%$ of elderly patients. ${ }^{11-19}$

Traditionally, a polyethylene glycol (PEG)-based preparation requires the ingestion of a large volume of liquid. However, the tolerability of a bowel preparation is related to its volume, taste, and adverse effects (which are particularly troublesome in elderly patients). Therefore, low-volume bowel cleansing agents are associated with a greater willingness to undergo repeat colonoscopy. ${ }^{20,21}$ A new oral sulfate solution (OSS) formulation with a split-dose regimen has been recently approved as a lowvolume regimen. ${ }^{7.8}$ Although a few studies have evaluated the

Correspondence to: Jae Myung Cha (https://orcid.org/0000-0001-9403-230X)

Department of Internal Medicine, Kyung Hee University Hospital at Gangdong, Kyung Hee University School of Medicine, 892 Dongnam-ro, Gangdong-gu, Seoul 05278, Korea

Tel: +82-2-440-6113, Fax: +82-2-440-6295, E-mail: drcha@khu.ac.kr

Received on May 14, 2018. Revised on August 3, 2018. Accepted on August 4, 2018. Published online January 3, 2019

pISSN 1976-2283 eISSN 2005-1212 https://doi.org/10.5009/gnl18214

(a) This is an Open Access article distributed under the terms of the Creative Commons Attribution Non-Commercial License (http://creativecommons.org/licenses/by-nc/4.0) which permits unrestricted non-commercial use, distribution, and reproduction in any medium, provided the original work is properly cited. 
efficacy and safety of OSS compared to those of PEG in average risk populations, data specific to the elderly population is lacking. ${ }^{7,8}$ Therefore, we conducted a multicenter, prospective, investigator-blinded, randomized, controlled trial (known as the SEESAFE study) to investigate the efficacy, safety, and acceptance of the OSS preparation with a split-dose regimen, in comparison to those of a standard PEG preparation, in elderly patients.

\section{MATERIALS AND METHODS}

\section{Eligibility criteria}

Participants aged 65 to 75 years who were willing to undergo colonoscopy were recruited between June 21, 2016 and July 7, 2017. Exclusion criteria were as follows: history of colorectal surgery; history of congestive heart failure or acute myocardial infarction within 6 months; American Society of Anesthesiologists physical status index $>3$; presence of liver cirrhosis, chronic renal failure, inflammatory bowel disease, or severe inflammation; severe constipation (bowel movement <3 times/week or use of stool softeners); a physical or mental disability that would interfere with participation; or refusal to provide consent. This study was approved by the local institutional review boards of all participating centers and the Institutional Review Board of Kyung Hee University Hospital at Gangdong (KHNMC IRB 2016-03-021). Written informed consent was obtained from all participants.

\section{Study design}

The SEE-SAFE study was a non-inferiority, prospective, randomized, investigator-blinded, multicenter study. The study was conducted at nine endoscopy centers in South Korea, and the trial was registered at Clinicaltrials.gov (identifier NCT03112967). Assignment to the 4-L PEG or OSS arm was determined according to a computer-generated randomization schedule, and participants were randomly allocated in a 1:1 ratio. Blood samples taken at the screening visit and the day of the procedure were analyzed for serum electrolytes (sodium, potassium, chloride, calcium, phosphate, and magnesium), blood urea nitrogen (BUN) level, creatinine level, and estimated glomerular filtration rate (GFR). The GFR was estimated using the Modification of Diet in Renal Disease Study equation (MDRD GFR). Acute kidney injury was defined as a 25\% increase in serum creatinine level. ${ }^{22}$

On the day of the procedure, all participants completed a questionnaire that collected the following information: taste, amount, and feeling sensations of the solution, acceptability, the amount of solution actually taken (complete/incomplete), adverse events (nausea, vomiting, abdominal pain, abdominal distension, fecal incontinence, sleep disturbance, numbness, general weakness, seizure, mental change, thirstiness, dizziness, and tingling sensations), and willingness to repeat the same bowel preparation medication if indicated in the future (Supplementary Material 1). Feeling sensation is defined as the texture of the preparation from intake until swallowing. Satisfaction ratings were given on a visual analog scale (according to a scale from 1 to 10 , higher scores denoting greater satisfaction); the patient was asked to place a mark corresponding to their satisfaction level. Tolerability and acceptability were assessed using the questionnaire data on symptoms occurring during preparation and difficulties in complying with the intake of the solution (Supplementary Material 1).

Bowel preparation efficacy was assessed using Boston Bowel Preparation Scale (BBPS) definitions. ${ }^{23}$ In the BBPS, a 10-point (0-9) summation score is used to assess the overall bowel preparation quality, and is based on the evaluation of three segments of the colon (right colon, transverse colon, and left colon) using a 3-point scoring system (0-3; 0=unprepared colon with mucosa not seen because of solid stool, $1=$ portion of colonic mucosa of the segment seen, but other areas not well seen due to staining, residual stool, and/or opaque liquid, $2=$ minor amount of residual staining, stool, and/or opaque liquid, but colonic mucosa of the segment seen well, and $3=$ entire colonic mucosa seen well with no residual staining, stool, or opaque liquid). An adequate bowel preparation was defined as a total BBPS score $\geq 6$ with all segment scores $\geq 2$, and an excellent cleansing was defined as a total score $>7$. At the end of the colonoscopy, the endoscopist recorded the examination start time, the use of sedation, cecal intubation, colonic mucosal abnormalities (including aphthous ulcer), and any adverse events.

\section{Study preparations}

All subjects were instructed to start a low-residue diet 48 hours prior to the colonoscopy and were restricted to a clear fluids diet 24 hours the colonoscopy for the bowel preparation. Both bowel preparation solutions were administered using a split-dose regimen. Participants in the 4-L PEG arm ingested the first $2 \mathrm{~L}$ between 18:00 and 20:00 on the day before the colonoscopy; the second $2 \mathrm{~L}$ was ingested between 07:00 and 09:00 on the day of the colonoscopy. Participants in the OSS arm were instructed to pour one $180-\mathrm{mL}$ bottle of the study medication into a provided 480-mL mixing cup, fill it with water, and drink the entire volume, followed by two additional $480-\mathrm{mL}$ portions of water (between 18:00 and 20:00 on the day before the colonoscopy). The second OSS dose was ingested at approximately 06:00 on the day of the colonoscopy, using the same formulation protocol. In both study arms, the second dose was taken within 4 hours before the scheduled time of the colonoscopy.

\section{Outcome assessment}

The primary outcome measures of the present study were the bowel cleansing efficacy and the secondary outcome measures were the frequency and severity of adverse events, and the acceptability score. 


\section{Sample size calculation}

The sample size required for at least 80\% power to detect a 15\% group difference in adequate bowel cleansing frequency with a one-sided significance level of 0.025 was estimated, with



Fig. 1. Patient selection flowchart

OSS, oral sulfate solution; PEG, polyethylene glycol.

Table 1. Baseline Characteristics

\begin{tabular}{lccc}
\hline \multicolumn{1}{c}{ Characteristics } & 4-L PEG (n=96) & OSS (n=97) & p-value \\
\hline Age, yr & $69.3 \pm 2.9$ & $68.6 \pm 2.9$ & 0.094 \\
Sex & & & 0.617 \\
Male & $46(47.9)$ & $43(44.3)$ & \\
Female & $50(52.1)$ & $54(55.7)$ & \\
Body mass index, $\mathrm{kg} / \mathrm{m}^{2}$ & & & 0.345 \\
$\geq 25$ & $40(41.7)$ & $34(35.1)$ & \\
$<25$ & $56(58.3)$ & $63(64.9)$ & \\
Indication of colonoscopy & & & 0.610 \\
Screening & $42(43.8)$ & $43(44.3)$ & \\
Surveillance & $36(37.5)$ & $31(32.0)$ & \\
Diagnostic & $18(18.7)$ & $23(23.7)$ & \\
Underlying disease & & & 0.206 \\
None & $33(34.4)$ & $43(44.3)$ & \\
Ischemic heart disease & $2(2.1)$ & $2(2.1)$ & \\
Hypertension & $29(30.2)$ & $28(28.9)$ & \\
Diabetes & $7(7.3)$ & $2(2.1)$ & \\
Hypertension + diabetes & $15(15.6)$ & $12(12.4)$ & \\
Dyslipidemia & $2(2.1)$ & $3(3.1)$ & \\
Other* & $8(8.3)$ & $7(7.1)$ & \\
\hline Da & & & \\
\hline
\end{tabular}

Data are presented as mean \pm SD or number (\%).

OSS, oral sulfate solution; PEG, polyethylene glycol.

*Parkinson disease, cardiac arrhythmia, and carotid artery stenosis. the assumption that the proportion of adequate bowel cleansing would be approximately 86\% for the PEG group. ${ }^{24,25}$ Considering an expected dropout rate of 10\%, the total number of participants was calculated to be 198 .

\section{Statistics}

Group differences were evaluated using two-tailed Student t-tests for continuous variables and two-tailed chi-square test or Fisher exact tests for categorical variables. For laboratory data, the statistical significance of the change from baseline was evaluated using paired t-tests. All p-values are two-tailed, and a $p$-value $<0.05$ was considered statistically significant. Statistical analyses were performed using SPSS software version 20.0 (IBM Corp., Armonk, NY, USA).

\section{RESULTS}

During the study period, 198 patients were screened, and 193 were finally randomized and analyzed (Fig. 1). The median age was 69.0 years (standard deviation, 2.9), and the male-to-female ratio was 0.8:1. The distribution of underlying diseases and the indications for colonoscopy were similar in the two groups (Table 1).

\section{Colon cleansing efficacy}

No group differences in overall efficacy were observed, with “adequate" preparations achieved in 95.9\% (93/97) and 94.8\% (91/96) of patients in the OSS and 4-L PEG groups, respectively ( $p=0.747$ ) (Table 2). However, the OSS group had significantly higher cleansing efficacy scores for both the entire colon $(7.9 \pm 1.3$ for OSS vs $7.4 \pm 1.3$ for 4 -L PEG, $p=0.010$ ) and the right colon $(2.6 \pm 0.5$ for OSS vs $2.3 \pm 0.5$ for $4-\mathrm{L}$ PEG, $\mathrm{p}=0.001)$. The cleansing scores for the transverse and left colon were comparable between groups.

Table 2. Bowel Cleansing Efficacy as Assessed by the Boston Bowel Preparation Scale

\begin{tabular}{lccc}
\hline \multicolumn{1}{c}{ Bowel cleansing } & OSS & 4-L PEG & p-value \\
\hline Boston Bowel Preparation Scale & & & \\
$\quad$ Total & $7.9 \pm 1.3$ & $7.4 \pm 1.3$ & 0.010 \\
$\quad$ Right colon & $2.6 \pm 0.5$ & $2.3 \pm 0.5$ & 0.001 \\
Transverse colon $^{\dagger}$ & $2.7 \pm 0.5$ & $2.6 \pm 0.5$ & 0.177 \\
Left colon $^{\ddagger}$ & $2.7 \pm 0.5$ & $2.6 \pm 0.7$ & 0.210 \\
Primary efficacy success & & & 0.747 \\
$\quad$ Adequate & $93(95.9)$ & $91(94.8)$ & \\
$\quad$ Inadequate & $4(4.1)$ & $5(5.2)$ & \\
\hline
\end{tabular}

Data are presented as mean \pm SD or number (\%).

OSS, oral sulfate solution; PEG, polyethylene glycol.

${ }^{*}$ Cecum and ascending colon; ${ }^{\dagger}$ Hepatic flexure, transverse colon, and splenic flexure; ${ }^{\ddagger}$ Descending colon, sigmoid colon, and rectum. 


\section{Clinical safety}

The mean intensity for each preparation-related clinical adverse event is shown in Table 3. Slightly more gastrointestinal complaints were reported in the OSS group than in the 4-L PEG group; however, all symptomatic events including nausea, vomiting, abdominal pain, abdominal distension, and fecal inconti-

Table 3. Clinical Safety Profiles

\begin{tabular}{lrrr}
\hline \multicolumn{1}{c}{ Clinical adverse events } & OSS & 4-L PEG & p-value \\
\hline Gastrointestinal event & & & \\
Nausea & $0.8 \pm 1.9$ & $0.6 \pm 1.7$ & 0.358 \\
Vomiting & $0.1 \pm 0.8$ & $0.3 \pm 1.3$ & 0.170 \\
Abdominal pain & $0.7 \pm 1.7$ & $0.5 \pm 1.7$ & 0.634 \\
Abdominal distension & $0.7 \pm 1.7$ & $0.5 \pm 1.7$ & 0.634 \\
Fecal incontinence & $0.7 \pm 1.7$ & $0.5 \pm 1.7$ & 0.634 \\
Aphthous ulceration on colonoscopy & $2(2.1)$ & $1(1.0)$ & 1.000 \\
Neurologic event & & & \\
Sleep disturbance & $0.5 \pm 1.6$ & $0.6 \pm 1.7$ & 0.864 \\
Numbness & $0.0 \pm 0.1$ & $0.1 \pm 0.5$ & 0.434 \\
General weakness & $0 \pm 0.0$ & $0 \pm 0.0$ & NA \\
Seizure & $0 \pm 0.0$ & $0 \pm 0.0$ & NA \\
Mental change & $0 \pm 0.0$ & $0 \pm 0.0$ & NA \\
Thirstiness & $0.8 \pm 1.9$ & $0.6 \pm 1.7$ & 0.358 \\
Dizziness & $0.0 \pm 0.1$ & $0.0 \pm 0.1$ & 1.000 \\
Tingling sensation & $0.0 \pm 0.1$ & $0.0 \pm 1.1$ & 1.000 \\
\hline
\end{tabular}

Data are presented as mean \pm SD or number (\%).

OSS, oral sulfate solution; PEG, polyethylene glycol; NA, not available. nence were rated as mild. In terms of neurologic symptomatic events, a slightly higher sleep disturbance score was observed in the 4-L PEG group than in the OSS group ( $p=0.864)$, while the OSS group reported slightly higher thirstiness scores $(p=0.358)$ (Table 3$)$. In addition, numbness scores were slightly more favorable in the OSS group than in the 4-L PEG group ( $p=0.434)$. However, there were no statistically significant group differences. None of the participants experienced seizures or mental changes (Table 3 ). None of the participants had a clinically significant need of intervention to manage the adverse events. All patients successfully completed intake of the whole preparation, and the procedure was performed without any problems.

\section{Renal and electrolyte safety}

The laboratory profile changes (on the procedure day vs baseline) for both groups are shown in Table 4. No serious adverse events were observed in the laboratory data. The groups were similar in the proportion of patients who had a $\geq 25 \%$ increase in creatinine relative to their baseline level $(2.1 \%$ in each group, $\mathrm{p}=1.000$ ) (Table 4). In both groups, serum BUN level significantly decreased after the bowel preparation without clinical significance; however, no significant changes were observed in serum creatinine levels (Table 4). In addition, there were no significant changes in the MDRD GFR (before OSS vs after OSS, 84.4 \pm 23.7 $\mathrm{mL} / \mathrm{min}$ vs $84.9 \pm 20.2 \mathrm{~mL} / \mathrm{min}, \mathrm{p}=0.744$; $4-\mathrm{L}$ PEG, $88.9 \pm 24.8$ $\mathrm{mL} / \mathrm{min}$ vs $87.2 \pm 22.9 \mathrm{~mL} / \mathrm{min}, \mathrm{p}=0.187$ ). In the 4 -L PEG group, serum calcium levels significantly decreased from $9.1 \pm 0.6$ to $9.0 \pm 0.5 \mathrm{mg} / \mathrm{L}$ after the bowel preparation $(\mathrm{p}=0.037)$. Statistically significant changes in serum sodium levels were shown in both groups following bowel preparation without clinical significance (OSS, $p=0.021$; 4-L PEG, $p=0.002$ ). In the OSS group, serum chlo-

Table 4. Laboratory Safety Profiles

\begin{tabular}{|c|c|c|c|c|c|c|c|}
\hline \multirow{2}{*}{ Variable } & \multicolumn{3}{|c|}{ OSS } & \multirow{2}{*}{$\mathrm{p}$-value } & \multicolumn{3}{|c|}{ 4-L PEG } \\
\hline & Before & $\mathrm{p}$-value & After & & Before & $\mathrm{p}$-value & After \\
\hline \multicolumn{8}{|l|}{ Laboratory results } \\
\hline \multicolumn{8}{|l|}{ Renal function profiles } \\
\hline BUN, mg/dL & $16.9 \pm 5.9$ & $<0.001$ & $14.5 \pm 4.6$ & & $14.9 \pm 5.0$ & $<0.001$ & $13.1 \pm 3.8$ \\
\hline Creatinine, mg/dL & $0.9 \pm 0.2$ & 0.205 & $0.9 \pm 0.2$ & & $0.9 \pm 0.3$ & 0.684 & $0.9 \pm 0.2$ \\
\hline MDRD GFR, $\mathrm{mL} / \mathrm{min}$ & $84.4 \pm 23.7$ & 0.744 & $84.9 \pm 20.2$ & & $88.9 \pm 24.8$ & 0.187 & $87.2 \pm 22.9$ \\
\hline AKI & & & $2(2.1)$ & 1.000 & & & $2(2.1)$ \\
\hline \multicolumn{8}{|l|}{ Electrolyte profiles } \\
\hline Calcium, mg/dL & $9.2 \pm 0.6$ & 0.502 & $9.2 \pm 0.5$ & & $9.1 \pm 0.6$ & 0.037 & $9.0 \pm 0.5$ \\
\hline Phosphate, mg/dL & $4.0 \pm 3.8$ & 0.177 & $3.5 \pm 0.6$ & & $3.5 \pm 0.5$ & 0.909 & $3.5 \pm 0.5$ \\
\hline Magnesium, mg/dL & $2.2 \pm 0.2$ & 0.068 & $2.2 \pm 0.2$ & & $2.2 \pm 0.3$ & 0.490 & $2.4 \pm 2.4$ \\
\hline Sodium, mg/dL & $141.3 \pm 3.0$ & 0.021 & $140.4 \pm 3.2$ & & $140.9 \pm 2.4$ & 0.002 & $141.8 \pm 2.5$ \\
\hline Potassium, mmol/L & $4.8 \pm 4.2$ & 0.829 & $4.6 \pm 3.8$ & & $4.7 \pm 4.2$ & 0.216 & $4.2 \pm 0.3$ \\
\hline Chloride, $\mathrm{mmol} / \mathrm{L}$ & $105.0 \pm 10.4$ & 0.030 & $102.6 \pm 3.4$ & & $103.0 \pm 10.9$ & 0.285 & $104.2 \pm 2.7$ \\
\hline
\end{tabular}

Data are presented as mean \pm SD or number (\%).

OSS, oral sulfate solution; PEG, polyethylene glycol; BUN, blood urea nitrogen; MDRD GFR, Modification of Diet in Renal Disease Study equation for estimated glomerular filtration rate; AKI, acute kidney injury. 
Table 5. Tolerability and Acceptability of the Preparation Agents

\begin{tabular}{|c|c|c|c|}
\hline Acceptability & OSS & 4-L PEG & p-value \\
\hline \multicolumn{4}{|l|}{ Satisfaction for the preparation } \\
\hline Taste & $6.2 \pm 1.9$ & $5.9 \pm 2.2$ & 0.328 \\
\hline Amount & $7.7 \pm 2.1$ & $6.4 \pm 2.8$ & $<0.001$ \\
\hline Feeling & $6.7 \pm 2.1$ & $5.8 \pm 2.5$ & 0.007 \\
\hline \multicolumn{3}{|l|}{ Willingness to repeat same preparation } & $<0.001$ \\
\hline Acceptable & $90(92.8)$ & $65(67.7)$ & \\
\hline Not acceptable & $7(7.2)$ & $31(32.3)$ & \\
\hline Amount of solution actually taken & & & 1.000 \\
\hline Complete & \multicolumn{3}{|c|}{97 (100.0) 96 (100.0) } \\
\hline Incomplete & 0 & 0 & \\
\hline Overall satisfaction & $7.7 \pm 1.5$ & $6.8 \pm 2.1$ & 0.001 \\
\hline
\end{tabular}

Data are presented as mean \pm SD or number (\%).

OSS, oral sulfate solution; PEG, polyethylene glycol.

ride levels significantly decreased from $105.0 \pm 10.4$ to $102.6 \pm 3.4$ $\mathrm{mmol} / \mathrm{L}$ after the bowel preparation $(\mathrm{p}=0.030)$. However, there were no clinically significant changes in any patients with changes in laboratory findings. Furthermore, there were no significant changes in serum phosphate, magnesium, and potassium levels following preparation administration in both groups (Table 4).

\section{Acceptability}

The groups did not remarkably differ in taste ratings $(\mathrm{p}=0.328)$ (Table 5); however, the preparation solution was easier to ingest in the OSS group due to its lower volume and higher "good feeling" ratings than in the 4-L PEG group ( $p<0.001, p=0.007$, respectively). Furthermore, significantly more patients indicated that they would be willing to use the same preparation in any future colonoscopies in the OSS group than in the 4-L PEG group (92.8\% vs 67.7\%, p<0.001). In addition, the overall satisfaction scores were significantly higher in the OSS group than in the 4 -L PEG group (7.7 \pm 1.5 vs $6.8 \pm 2.1, \mathrm{p}=0.001$ ) (Table 5).

\section{DISCUSSION}

Age has been consistently associated with poor bowel preparation in previous studies, regardless of the type of preparation. ${ }^{26-29}$ A recent systematic review and meta-analysis reported that nearly 20\% of patients aged 65 years or older have poor bowel preparation at the time of their colonoscopy. ${ }^{30}$ Therefore, improving bowel preparation in this age group is an important issue. The results of the present investigator-blinded, prospective, multicenter, randomized clinical trial demonstrate that the OSS preparation is not inferior (in terms of efficacy, safety, and tolerability) to the 4-L PEG preparation in elderly patients. This is the first report to specifically analyze the efficacy, safety, and acceptability of the new OSS preparation in elderly individuals, and provide information important to the selection of the proper bowel preparation protocol for this population.
While 4-L PEG is the most commonly used bowel preparation regimen because of its efficacy and safety properties, the main disadvantage, especially for the elderly, is the substantial volume that must be ingested. However, many clinicians are reluctant to prescribe small volume preparations for elderly patients, as elderly patients are more likely to have poor bowel preparation and are more vulnerable to clinical and laboratory adverse events. Although a previous multicenter study in the US $(n=136)$ reported that OSS ingestion more frequently resulted in an excellent preparation compared to 4-L PEG ingestion (71.4\% vs $34.3 \%$ ), the study included only 29 elderly patients ( $\geq 65$ years old). ${ }^{8}$ In addition, the study design was not fair as the OSS was administered in split regimen, whereas PEG was administered in a non-split regimen. ${ }^{8}$ Thus, the efficacy of the OSS preparation in the elderly population was not well addressed. Of note, the results for the primary efficacy end point in the present study confirmed that, in elderly patients, OSS and 4-L PEG preparations result in similar cleansing efficacy, with "adequate" preparations achieved in 95.5\% of OSS patients and 95.2\% of 4-L PEG patients. More importantly, we found that the OSS preparation achieved better cleansing scores than the 4-L PEG preparation in the entire and right colon. CRC in elderly patients more often involves the right colon and grows faster than in younger patients. ${ }^{31,32}$ However, the cleanliness of the right colon is often unsatisfactory with low volume preparations. ${ }^{33,34}$ Thus, the present results add to the literature, suggesting that OSS preparation with a split-dose regimen better prepares the right colon for evaluation and may be more effective in CRC screening in elderly patients than the 4-L PEG preparation.

Theoretically, the osmotic action of the poorly absorbed sulfates and magnesium in the OSS formulation increases the water content of the stool, thus causing watery diarrhea, which can result in volume depletion. Although OSS is considered safe as a bowel purgative based on animal ${ }^{35}$ and young adult human studies, ${ }^{36}$ limited data currently exist regarding the safety of OSS in terms of renal toxicity and electrolyte disturbance, especially in elderly patients who may be more vulnerable to dehydration than younger patients. Accordingly, concerns exist regarding the occurrence of such adverse events during OSS bowel preparation in elderly individuals. However, the present results indicate that the changes in serum creatinine levels and MDRD GFRs after preparation were similar for OSS and 4-L PEG. In the present study, OSS bowel preparation resulted in a statistically significant decrease in serum chloride values; however, these differences were small and no clinically significant complications were reported. In addition, both preparations were associated with clinically insignificant changes in serum BUN and electrolyte profiles. Furthermore, clinical adverse events were not significantly different between the two preparations, and no serious adverse events such as seizure, mental change, or death were reported. These results are also comparable to those reported in previous studies. For example, in a previous 
US study, the overall and gastrointestinal discomfort scores for OSS with a split-dose regimen were similar to those for 4-L PEG in patients aged 65 years or older. ${ }^{7}$ In addition, Rex et al. ${ }^{8}$ reported that there were no differences between OSS and 4-L PEG preparations in terms of treatment-emergent adverse events in the elderly group. Taken together, these results suggest OSS may be considered as an appropriate option for bowel cleansing in elderly patients.

Another noteworthy finding in the present study concerns the acceptability of the OSS preparation. Compared to 4-L PEG, OSS resulted in better scores regarding the sensation of the amount and feeling of the preparation, which can be potential barriers to future colonoscopy acceptance in elderly patients. Further, the overall satisfaction scores were significantly higher with the OSS preparation than with the 4-L PEG preparation, and patients who received the OSS preparation were more likely to be willing to repeat the preparation in the future than were patients who received the 4-L PEG preparation. In summary, the OSS preparation with a split-dose regimen achieves greater bowel cleansing efficacy, with a similar safety profile and acceptability, than a standard regimen of 4-L PEG in elderly patients.

The present study has some limitations that warrant consideration. The preparation quality was evaluated by different endoscopists; thus, inter- or intra-observer differences may have introduced biases. However, previous studies have consistently demonstrated that the BBPS is a reliable measure for preparation quality, with good inter- and intra-observer reliability among physicians, ${ }^{23,37}$ further all study investigators reviewed the representative captured images and reached a consensus before study enrollment. to achieve better inter-observer agreement. Another limitation concerns the limited generalizability of the present findings to Western populations given the exclusive recruitment of Asian individuals in the present study. Therefore, further studies with different ethnic populations are needed. In addition, the present study included only elderly patients aged 65 to 75 and our sample size was too small to provide conclusive data on the safety and efficacy of OSS. To confirm these findings, further large-scaled studies with very elderly ( $\geq 75$ years old) patients are warranted. Finally, unmeasured several confounders including prior experience of bowel preparation that may also contribute to the results.

In conclusion, the present study demonstrates that OSS, a low-volume bowel preparation solution, is efficacious and safe bowel cleansing tool in elderly individuals. Moreover, the use of OSS enables future colonoscopy acceptability in this population. Further studies with larger sample sizes and different ethnic populations are warranted to confirm these results.

\section{CONFLICTS OF INTEREST}

No potential conflict of interest relevant to this article was reported.

\section{ACKNOWLEDGEMENTS}

This study was funded by TAEJOON PHARM Co., Ltd, Inc. Author contributions: Concept and design: M.S.K. and J.M.C.; Acquisition of data: H.J.Y., D.I.P., K.O.K., J.L., J.E.S., Y.E.J., J.P., J.S.B., and H.G.K.; Statistical analysis: M.S.K. and J.M.C.; Drafting and revision of manuscript: M.S.K. and J.M.C.

\section{REFERENCES}

1. Rabeneck L, El-Serag HB, Davila JA, Sandler RS. Outcomes of colorectal cancer in the United States: no change in survival (1986-1997). Am J Gastroenterol 2003;98:471-477.

2. Jemal A, Siegel R, Xu J, Ward E. Cancer statistics, 2010. CA Cancer J Clin 2010;60:277-300.

3. Doubeni CA, Corley DA, Quinn VP, et al. Effectiveness of screening colonoscopy in reducing the risk of death from right and left colon cancer: a large community-based study. Gut 2018;67:291298.

4. Nishihara R, Wu K, Lochhead P, et al. Long-term colorectal-cancer incidence and mortality after lower endoscopy. N Engl J Med 2013;369:1095-1105.

5. Lieberman DA, Rex DK, Winawer SJ, Giardiello FM, Johnson DA, Levin TR. Guidelines for colonoscopy surveillance after screening and polypectomy: a consensus update by the US Multi-Society Task Force on Colorectal Cancer. Gastroenterology 2012;143:844857.

6. Rex DK, Johnson DA, Anderson JC, et al. American College of Gastroenterology guidelines for colorectal cancer screening 2009 [corrected]. Am J Gastroenterol 2009;104:739-750.

7. Di Palma JA, Rodriguez R, McGowan J, Cleveland Mv. A randomized clinical study evaluating the safety and efficacy of a new, reduced-volume, oral sulfate colon-cleansing preparation for colonoscopy. Am J Gastroenterol 2009;104:2275-2284.

8. Rex DK, Di Palma JA, Rodriguez R, McGowan J, Cleveland M. A randomized clinical study comparing reduced-volume oral sulfate solution with standard 4-liter sulfate-free electrolyte lavage solution as preparation for colonoscopy. Gastrointest Endosc 2010;72:328-336.

9. Katz P0, Rex DK, Epstein M, et al. A dual-action, low-volume bowel cleanser administered the day before colonoscopy: results from the SEE CLEAR II study. Am J Gastroenterol 2013;108:401409.

10. Rex DK, Katz PO, Bertiger G, et al. Split-dose administration of a dual-action, low-volume bowel cleanser for colonoscopy: the SEE CLEAR I study. Gastrointest Endosc 2013;78:132-141.

11. Karajeh MA, Sanders DS, Hurlstone DP. Colonoscopy in elderly people is a safe procedure with a high diagnostic yield: a prospective comparative study of 2000 patients. Endoscopy 2006;38:226230.

12. Ma WT, Mahadeva S, Kunanayagam S, Poi PJ, Goh KL. Colonoscopy in elderly Asians: a prospective evaluation in routine clinical 
practice. J Dig Dis 2007;8:77-81.

13. Lukens FJ, Loeb DS, Machicao VI, Achem SR, Picco MF. Colonoscopy in octogenarians: a prospective outpatient study. Am J Gastroenterol 2002;97:1722-1725.

14. Bat L, Pines A, Shemesh E, et al. Colonoscopy in patients aged 80 years or older and its contribution to the evaluation of rectal bleeding. Postgrad Med J 1992;68:355-358.

15. Burtin P, Bour B, Charlois T, et al. Colonic investigations in the elderly: colonoscopy or barium enema? Aging (Milano) 1995;7:190194.

16. Chatrenet P, Friocourt P, Ramain JP, Cherrier M, Maillard JB. Colonoscopy in the elderly: a study of 200 cases. Eur J Med 1993;2:411-413.

17. Schmilovitz-Weiss H, Weiss A, Boaz M, Levin I, Chervinski A, Shemesh E. Predictors of failed colonoscopy in nonagenarians: a single-center experience. J Clin Gastroenterol 2007;41:388-393.

18. Duncan JE, Sweeney WB, Trudel JL, Madoff RD, Mellgren AF. Colonoscopy in the elderly: low risk, low yield in asymptomatic patients. Dis Colon Rectum 2006;49:646-651.

19. Syn WK, Tandon U, Ahmed MM. Colonoscopy in the very elderly is safe and worthwhile. Age Ageing 2005;34:510-513.

20. DiPalma JA, Wolff BG, Meagher A, Cleveland Mv. Comparison of reduced volume versus four liters sulfate-free electrolyte lavage solutions for colonoscopy colon cleansing. Am J Gastroenterol 2003;98:2187-2191.

21. Ell C, Fischbach W, Bronisch HJ, et al. Randomized trial of lowvolume PEG solution versus standard PEG + electrolytes for bowel cleansing before colonoscopy. Am J Gastroenterol 2008;103:883893.

22. Brunelli SM, Lewis JD, Gupta M, Latif SM, Weiner MG, Feldman HI. Risk of kidney injury following oral phosphosoda bowel preparations. J Am Soc Nephrol 2007;18:3199-3205.

23. Lai EJ, Calderwood AH, Doros G, Fix OK, Jacobson BC. The Boston bowel preparation scale: a valid and reliable instrument for colonoscopy-oriented research. Gastrointest Endosc 2009;69(3 Pt 2):620-625.

24. Schanz S, Kruis W, Mickisch 0, et al. Bowel preparation for colonoscopy with sodium phosphate solution versus polyethylene glycol-based lavage: a multicenter trial. Diagn Ther Endosc 2008;2008:713521.
25. Kojecky V, Dolina J, Kianicka B, et al. A single or split dose picosulphate/magnesium citrate before colonoscopy: comparison regarding tolerance and efficacy with polyethylene glycol. A randomized trial. J Gastrointestin Liver Dis 2014;23:141-146.

26. Chung YW, Han DS, Park KH, et al. Patient factors predictive of inadequate bowel preparation using polyethylene glycol: a prospective study in Korea. J Clin Gastroenterol 2009;43:448-452.

27. Cha JM. Would you recommend screening colonoscopy for the very elderly? Intest Res 2014;12:275-280.

28. Cha JM, Kozarek RA, La Selva D, et al. Risks and benefits of colonoscopy in patients 90 years or older, compared with younger patients. Clin Gastroenterol Hepatol 2016;14:80-86.

29. Loffeld RJ, Liberov B, Dekkers PE. Yearly diagnostic yield of colonoscopy in patients age 80 years or older, with a special interest in colorectal cancer. Geriatr Gerontol Int 2012;12:298-303.

30. Day LW, Kwon A, Inadomi JM, Walter LC, Somsouk M. Adverse events in older patients undergoing colonoscopy: a systematic review and meta-analysis. Gastrointest Endosc 2011;74:885-896.

31. Patel SS, Nelson R, Sanchez J, et al. Elderly patients with colon cancer have unique tumor characteristics and poor survival. Cancer 2013;119:739-747.

32. Holt PR, Kozuch P, Mewar S. Colon cancer and the elderly: from screening to treatment in management of GI disease in the elderly. Best Pract Res Clin Gastroenterol 2009;23:889-907.

33. Berkelhammer C, Ekambaram A, Silva RG. Low-volume oral colonoscopy bowel preparation: sodium phosphate and magnesium citrate. Gastrointest Endosc 2002;56:89-94.

34. Corporaal S, Kleibeuker JH, Koornstra JJ. Low-volume PEG plus ascorbic acid versus high-volume PEG as bowel preparation for colonoscopy. Scand J Gastroenterol 2010;45:1380-1386.

35. Pelham RW, Russell RG, Padgett EL, Reno FE, Cleveland Mv. Safety of oral sulfates in rats and dogs contrasted with phosphateinduced nephropathy in rats. Int J Toxicol 2009;28:99-112.

36. Patel V, Nicar M, Emmett M, et al. Intestinal and renal effects of low-volume phosphate and sulfate cathartic solutions designed for cleansing the colon: pathophysiological studies in five normal subjects. Am J Gastroenterol 2009;104:953-965.

37. Calderwood AH, Jacobson BC. Comprehensive validation of the Boston Bowel Preparation Scale. Gastrointest Endosc 2010;72:686692. 